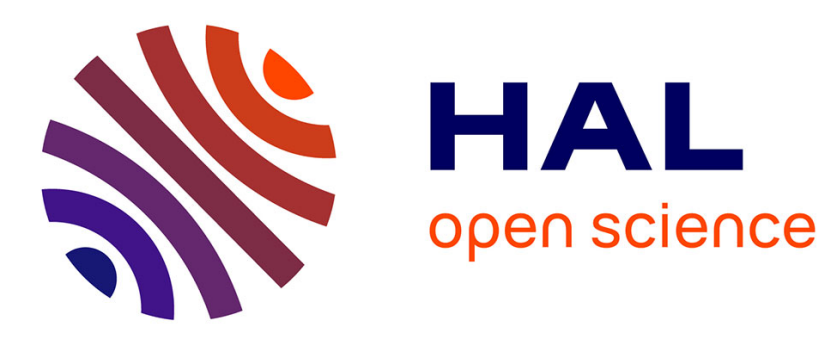

\title{
Taking advantage of acoustic inhomogeneities in photoacoustic measurements
}

Anabela da Silva, Charles Handschin, Christophe Riedinger, Julien Piasecki, Serge Mensah, Amélie Litman, Hassan Akhouayri

\section{- To cite this version:}

Anabela da Silva, Charles Handschin, Christophe Riedinger, Julien Piasecki, Serge Mensah, et al.. Taking advantage of acoustic inhomogeneities in photoacoustic measurements. SPIE BiOS, Feb 2016, San Francisco, United States. pp.97082B, 10.1117/12.2209549 . hal-03515259

\author{
HAL Id: hal-03515259 \\ https://hal.science/hal-03515259
}

Submitted on 6 Jan 2022

HAL is a multi-disciplinary open access archive for the deposit and dissemination of scientific research documents, whether they are published or not. The documents may come from teaching and research institutions in France or abroad, or from public or private research centers.
L'archive ouverte pluridisciplinaire $\mathbf{H A L}$, est destinée au dépôt et à la diffusion de documents scientifiques de niveau recherche, publiés ou non, émanant des établissements d'enseignement et de recherche français ou étrangers, des laboratoires publics ou privés. 


\title{
Taking advantage of acoustic inhomogeneities in photoacoustic measurements
}

\author{
Anabela Da Silva ${ }^{\mathrm{a}}$, Charles Handschin ${ }^{\mathrm{a}, \mathrm{b}}$, Christophe Riedinger ${ }^{\mathrm{a}, \mathrm{b}}$, Julien Piasecki ${ }^{\mathrm{b}}$, Serge \\ Mensah $^{\mathrm{c}}$, Amélie Litman ${ }^{\mathrm{a}}$, and Hassan Akhouayri ${ }^{\mathrm{a}}$ \\ ${ }^{a}$ Aix-Marseille Université, CNRS, Centrale Marseille, Institut Fresnel UMR 7249, 13013 \\ Marseille, France

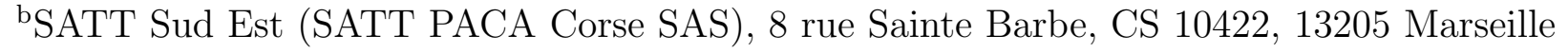 \\ Cedex 01, France \\ cAix-Marseille Université, CNRS, CNRS, Centrale Marseille, LMA UPR 7051, 13402 Marseille \\ Cedex 20, France
}

\begin{abstract}
Photoacoustic offers promising perspectives in probing and imaging subsurface optically absorbing structures in biological tissues. The optical fluence absorbed is partly dissipated into heat accompanied with microdilatations that generate acoustic pressure waves, the intensity which is related to the amount of fluence absorbed. Hence the photoacoustic signal measured offers access, at least potentially, to a local monitoring of the absorption coefficient, in 3D if tomographic measurements are considered. However, due to both the diffusing and absorbing nature of the surrounding tissues, the major part of the fluence is deposited locally at the periphery of the tissue, generating an intense acoustic pressure wave that may hide relevant photoacoustic signals. Experimental strategies have been developed in order to measure exclusively the photoacoustic waves generated by the structure of interest (orthogonal illumination and detection). Temporal or more sophisticated filters (wavelets) can also be applied. However, the measurement of this primary acoustic wave carries a lot of information about the acoustically inhomogeneous nature of the medium. We propose a protocol that includes the processing of this primary intense acoustic wave, leading to the quantification of the surrounding medium sound speed, and, if appropriate to an acoustical parametric image of the heterogeneities. This information is then included as prior knowledge in the photoacoustic reconstruction scheme to improve the localization and quantification.
\end{abstract}

Keywords: Photoacoustic imaging, Medical and biological imaging, Inverse problems

\section{INTRODUCTION}

The first goal of Photoacoustic tomography is to provide indirectly, by the resolution of the inverse problem, a measurement of the optical absorption coefficient of heterogeneously absorbing media. Quantification of this absorption coefficient can be obtained if the physics of the optical diffusion process is also considered in the model, and a discrimination between absorption and diffusion is achieved. Interrogating the medium with multiple sources illumination ${ }^{1}$ is a method that allows this. Local illumination produce a primary intense photoacoustic wave, localized in a volume close to the interface. Usually, one seeks at positioning sources and tranducers in a geometric way such that this signal doesn't affect much the measures of the photoacoustic signal coming from the inhomogeneity of interest. However, this primary intense photoacoustic wave, propagating through the medium, can serve as an acoutic probe sensitive to the acoustic inhomogeneities present in the medium, like tumors, heterogeneous both optically (neo-angiogenesis) and acoustically (detected with conventional ultrasound echography). With local sources illumination, the interesting point is that the detection of this acoustically sensitive signal and the more conventional photoacoustic signal is detected within two separate times intervals. A classical time filter can be applied to separate in time the two species of signals, that can afterwards be exploited separatly: the acoustic echoes bring spatial information that can be introduced as prior knowledge in the

Further author information: (Send correspondence to Anabela Da Silva)

Anabela Da Silva: E-mail: anabela.dasilva@fresnel.fr, Telephone: +3349128 8482 
photoacoustic signal processing to retrieve the optical properties. In the present work, through a demonstrating experimental photoacoustic measurement performed on a phantom presenting an optical/acoutic inhomogeneity, the information content of the acquired signals is presented and dicussed, and the filtering protocol to exploit the acoustic information extracted from the measured photoacoustic signal is illustrated through simulations performed on a synthetic phantom.

\section{EXPERIMENTAL DEMONSTRATION}

In photoacoustics, the medium is heated with a time varying optical source. The thermal expansion of the tissues generates acoustic pressure waves that can be measured with transducers. Sound and light velocities being so different, the optical fluence is going to be deposited instantaneously within the whole medium according to the spatial distribution of the absorption and the diffusion coefficients of the probed tissue. The deposited energy is dissipated into heat, microdilatations are produced that give birth to a pressure wave that can propagate through the medium. If the medium is homogeneous in terms of both optical and acoustic properties, a single acoustic wave pressure echo, is going to be measured due to the deposition of energy, mostly near the illuminated surface because of the short scattering mean free path (few tens $\mu \mathrm{m}$ in biological tissues). Hence its time-offlight corresponds roughly to the distance between the illuminated surface and the detector position. However, the fact is that this initial pressure wave is going to propagate and potentially being able probe the medium as a conventional acoustic wave. Hence, if acoustic heterogeneities are present in the medium, for example because of a different density as in tumors (mammary tumors are visible with US echography), then this primary photoacoustic wave is going to be perturbed, and echoes related to the presence of acoustic heterogenities will be sensed at longer times-of-flight. If optically absorbing heterogeneities are present within the medium, more energy will be absorbed locally giving birth to the conventional photoacoustic echoes. In order to eyelight this phenomenon, hereafter is shown a measurement performed on an Agar-Agar based phantom. Our setup (Fig.1, Left) comprises a pulsed laser (Quantel, France, Nd:Yag, $1064 \mathrm{~nm}, 330 \mathrm{~mJ}, 5 \mathrm{~ns}, 10 \mathrm{~Hz}$, frequency doubled by a KTP crystal at $532 \mathrm{~nm}$ ) and with immersion acoustic transducers (diameter $10 \mathrm{~mm}$, focal length 1, central frequency $3.5 \mathrm{MHz}$, Olympus, France) are used. The illumination beam was enlarged in the transverse direction (size of the beam $40 \mathrm{~mm}$ ). The collected signal is then preamplified (Olympus, France, gain $40 \mathrm{~dB}$, cutting frequency $10 \mathrm{MHz}$ ) and drived to an oscilloscope (Tektronix, USA, 4 channels, bandpass width $200 \mathrm{MHz}$ ) for signal analyzis.
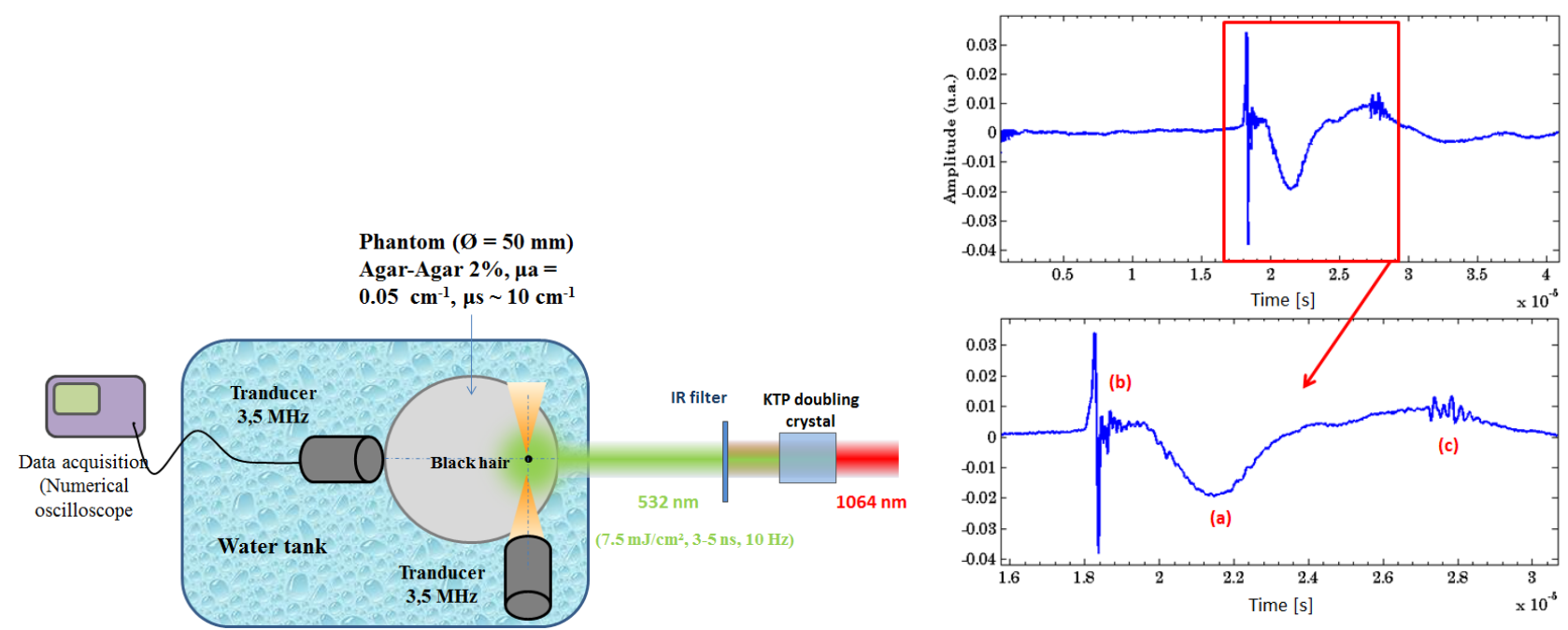

Figure 1. Left:Schema of the experimental setup and phantom; Right: Phantom measurement with hair inclusion: (a) photoacoustic response of the surface, (b) photoacoustic response of the inclusion, (c) acoustic response of the inclusion.

The phantom is a cylinder $(\varnothing=5 \mathrm{~cm})$ made of a aqueous gels of a mixture of agar-agar (volume fraction $2 \%$, Jeulin, France) and titanium dioxyde $\left(\mathrm{TiO}_{2}\right)$ powder $(0.05 \%$, Sigma-Aldrich, France) to mimick the level of optical scattering of the tissues (reduced scattering coefficient estimated $6 \mathrm{~cm}^{-1}$, and absorption coefficient 
$0.02 \mathrm{~cm}^{-1}$ at $\left.532 \mathrm{~nm}\right)$. The inclusion is a black hair placed as in Fig.1, Left. The measurement, collected with a transducer placed as in Fig.1, Left, perpendicular to the illumination path, is plotted on (Fig.1, Right).

The photoacoustic echo arrives approximately at $t_{i} \sim 18 \mu \mathrm{s}$, corresponding to the distance inclusiontransducer. As the phantom contains an acoustic heterogeneity, it can be orbserved the presence of a second echo, arriving at a time-of-flight corresponding to the path source-inclusion-tranducer: $t_{s i}+t_{i d} \sim 28 \mu \mathrm{s}$. This secondary acoustic echo, due to the presence of a burried structure acoustically inhomogeneous, allows retrieving spatial information on its location. This information can afterwards included in the reconstruction of the optical parameters as prior information to improve the inversion process.

Two echoes of different nature are present in the measurements: the photoacoustic ones (Fig.1, Right, (b)) and the acoustic ones (Fig.1, Right, (c)). The main difficulty lies in sorting temporally these signals of different nature. One can note that the two species of echoes belong to two different time spaces, delimited by the position of the interface (Fig.1, Right, (a)): (i) photoacoustic echo (b) is produced by a perturbating object $T$ belonging to a circle $C$ of radius $\overline{T D}=t_{(b)} / v_{s}, v_{s}$ being the sound velocity; (ii) acoustic echo (c) senses the presence of an object $T$ belonging to an ellipse $E$ of foci the position of the detector $\mathrm{D}$ and the projection of the laser beam at the surface of the phantom such that $\overline{S T}+\overline{T D}=t_{(c)} / v_{s}$. The perturbation $T$ belongs to the intersection of $C$ and $E$ within the phantom area. For multiple objects localization, one has to multiply the measurements and plot all possible occurences, as illustrated in the next section.

\section{ILLUSTRATION WITH SYNTHETIC DATA}

A 2D example of simulations explains how this measure can be exploited and introduced in a reconstruction algorithm to retrieve the optical parameters. A homogeneous disk phantom is considered containing two inhomogeneous regions, one disk (diameter $5 \mathrm{~mm}$ ) and one square $\left(2.5 \times 2.5 \mathrm{~mm}^{2}\right)$. The geometry is shown on Fig. 2. The following table lists the values of the physical parameters.

Table 1. Physical properties of the synthetic phantom.

\begin{tabular}{|c|c|c|c|}
\hline & Backgound & Disk inclusion & Square inclusion \\
\hline Absorption coefficient $\left[\mathrm{cm}^{-1}\right]$ & 0.1 & 0.11 & 0.1 \\
\hline Diffusion coefficient $[\mathrm{cm}]$ & 0.0333 & 0.0303 & 0.0303 \\
\hline Density $\left[\mathrm{kg} \cdot \mathrm{m}^{-3}\right]$ & 1000 & 1500 & 1700 \\
\hline Sound velocity $\left[\mathrm{m} . \mathrm{s}^{-1}\right]$ & 1485 & 1485 & 1485 \\
\hline
\end{tabular}

Synthetic pressure measurements were simulated by, first, computing for each source the initial pressure distribution $p_{o s}(\mathbf{r})=\Gamma(\mathbf{r}) H_{s}(\mathbf{r}), \Gamma$ [-] being the Grueneisen coefficient, $H_{s}(\mathbf{r})=\mu_{a}(\mathbf{r}) \phi_{s}(\mathbf{r})\left[\mathrm{W} . \mathrm{cm}^{-3}\right]$ the absorbed power density transformed into heat at position $\mathbf{r}, \mu_{a}(\mathbf{r})\left[\mathrm{cm}^{-1}\right]$ the optical absorption coefficient, $\phi_{s}(\mathbf{r})$ $\left[\mathrm{W} . \mathrm{cm}^{-2}\right]$ the fluence deposited by source $s$, calculated by solving numerically (Finite Element Method) the Diffusion Equation in the Continuous Wave regime. The k-wave toolbox were used to solve the acoustic propagation. ${ }^{2}$ In the present example, 360 transducers were evenly distributed at the periphery of the object and a set of 8 different point sources experiments (also evenly distributed) were run. The final sinogram (Fig. 2, $2^{\text {nd }}$ image) was composed of $8 \times 360$ time-resolved mesurements, the contribution of the homogeneous background was subtracted, an example of these measurements is plotted on Fig. 2 ( $3^{\text {rd }}$ image), showing the complexity of the signals. From the sinograms, an algorithm of peak detections is applied, ${ }^{3}$ and the spatial histogram of probabilities of intersections between circle $C$ and $E$ is calculated. The gradient of the resulting image is taken to obtained enhanced contours and a final thresholding allows rejecting artifacts due to sources positions (Fig. $2\left(4^{\text {th }}\right.$ image $\left.)\right)$.

A minimization based approach has been adopted for the resolution of the inverse problem, in which it is sought to find $\left(\mu_{a}, D\right)$ which minimizes the error functional $F=\frac{1}{2} \sum_{s} \int_{\Omega}\left(H_{s}^{m}(\mathbf{r})-H_{s}\left(\mathbf{r}, \mu_{a}, D\right)\right)^{2} d \mathbf{r}$, where $H_{s}^{m}$ is the measured initial pressure distribution map due to source $s$, that can be obtained from the measured pressures, after resolution of the acoustic propagation inverse problem. The reconstructions of the optical parameters have 

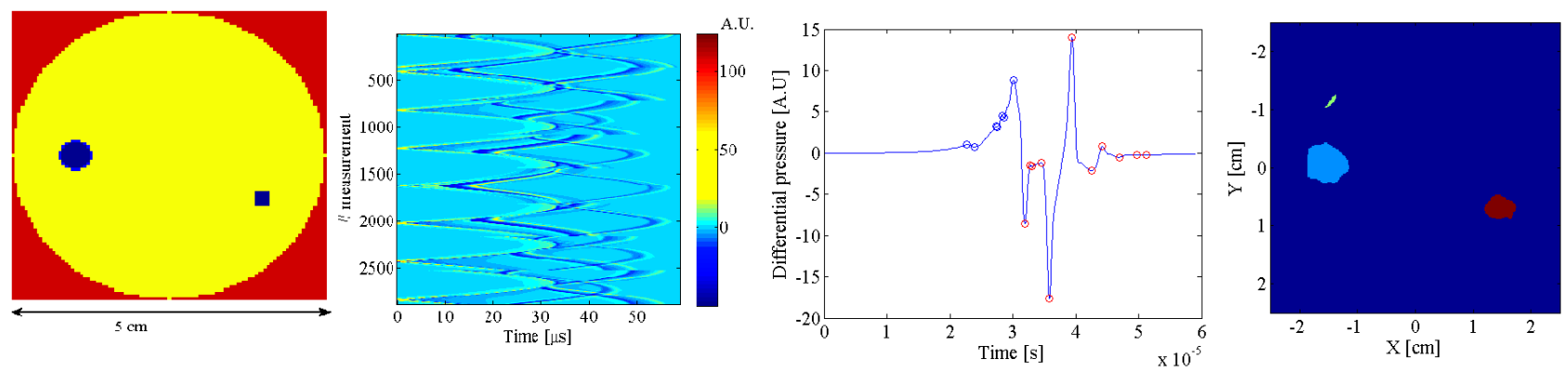

Figure 2. $1^{\text {st }}$ image: geometry, $2^{\text {nd }}$ : differential sinogram, $3^{\text {rd }}$ : measurement number 145 with peak detection (blue circles: photoacoustic peaks, read circles: acoustic peaks), $4^{\text {th }}$ : resulting map of probability of presence of inhomogeneities.

been performed with an adjoint-assisted gradient-based method. ${ }^{4}$ The reconstructions obtained with and without use of the localization map are shown on Fig. 3.
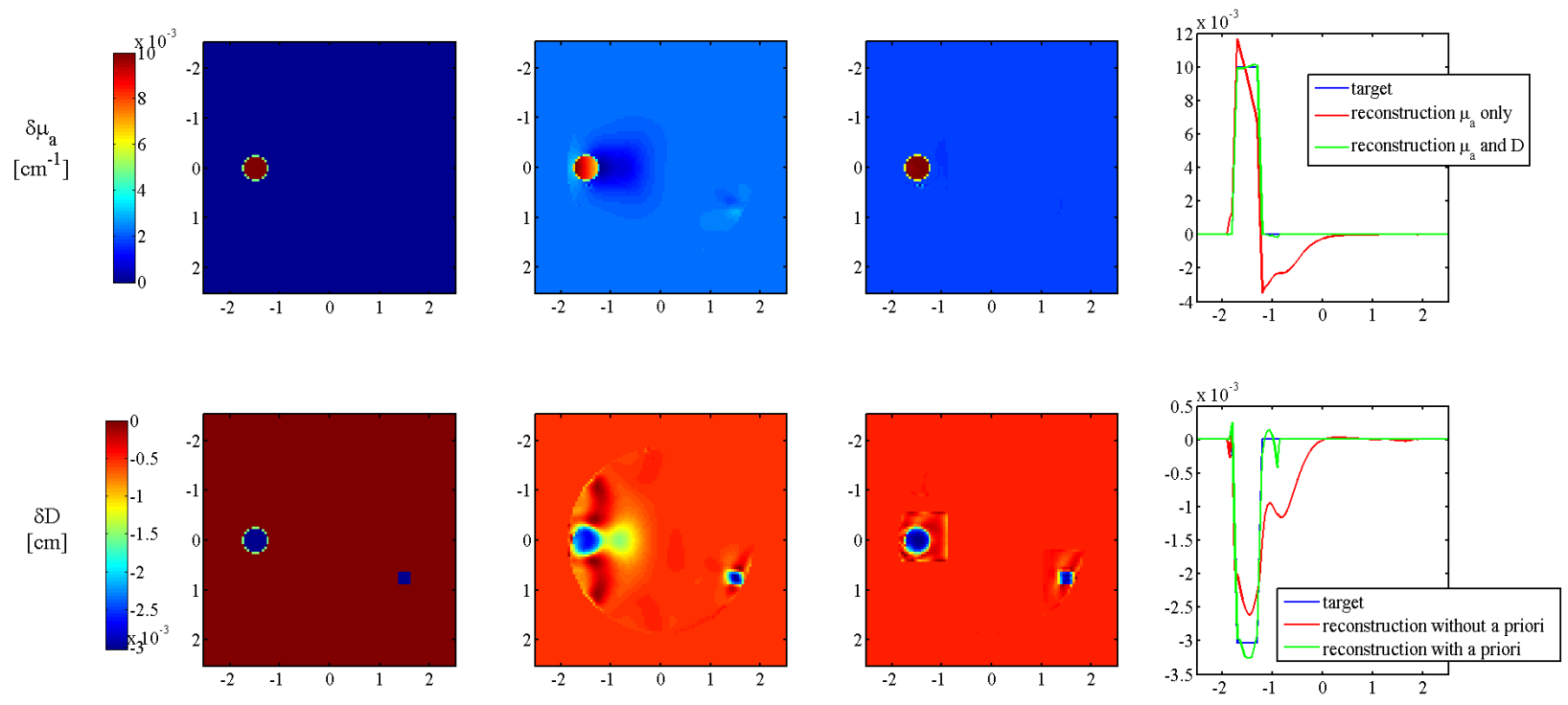

Figure 3. Optical parameters reconstructions. Top: absorption coefficient, target values, reconstruction of $\mu_{a}$ alone without prior, reconstruction of $\mu_{a}$ by simultaneously reconstruction $\mathrm{D}$, without prior, horizontal crossplot (Y=0). Bottom: diffusion coefficient, target values, reconstruction of $\mathrm{D}$ simulatneously with $\mu_{a}$, without prior, reconstruction of $\mathrm{D}$ simulatneously with $\mu_{a}$ with prior, horizontal crossplot $(\mathrm{Y}=0)$.

\section{CONCLUSION}

A demonstration experiment performed on a phantom with physical properties close to those of biological tissues shows the effect of perturbation of acoustic nature on the photoacoustic measurements. The specific measurement of this perturbation can be used to retrieve morphological information on the sample that can be used afterwards to constrain the reconstruction of the optical parameters. This is illustrated through an example of reconstruction performed on synthetic data.

\section{ACKNOWLEDGMENTS}

Agence National pour la Recherche (ANR) (AVENTURES-ANR-12-BLANBS01-0001-04); SATT-SE programme de maturation Mammoscan. 


\section{REFERENCES}

[1] Bal, G. and Ren, K., "Multi-source quantitative photoacoustic tomography in a diffusive regime," Inverse Problems 27, 075003,20p (2011).

[2] Cox, B. T., Kara, S., Arridge, S. R., and Beard, P. C., "k-space propagation models for acoustically heterogeneous media: Application to biomedical photoacoustics," Journal of the Acoustical Society of America 121, 3453-3464 (2007).

[3] Yoder, N. C., "Matlab function 'peakfinder.m'." //http://www.mathworks.com/matlabcentral/ fileexchange/25500-peakf inder-x0--sel--thresh--extrema--includeendpoints--interpolate-. (Copyright (c) 2015).

[4] Paul C. Beard, Jan G. Laufer, B. C. and Arridge, S. R., "Quantitative photoacoustic imaging: Measurement of absolute chromophore concentrations for physiological and molecular imaging," in [Photoacoustic Imaging and Spectroscopy], Wang, L. V., ed., CRC Press (2009). 